\title{
TORSION THEORIES OVER SEMIHEREDITARY RINGS
}

\author{
M. W. EVANS \\ (Received 23 August 1984) \\ Communicated by $\mathrm{H}$. Lausch
}

\begin{abstract}
In this paper the class of rings for which the right flat modules form the torsion-free class of a hereditary torsion theory $\left(\mathscr{T}_{G}, \mathscr{F}_{G}\right)$ are characterized and their structure investigated. These rings are called extended semihereditary rings. It is shown that the class of regular rings with ring homomorphism is a full co-reflective subcategory of the class of extended semihereditary rings with "flat" homomorphisms. A class of prime torsion theories is introduced which determines the torsion theory $\left(\mathscr{T}_{G}, \mathscr{F}_{G}\right)$. The torsion theory $\left(\mathscr{T}_{G}, \mathscr{F}_{G}\right)$ is used to find a suitable generalisation of Dedekind Domain.
\end{abstract} 1980 Mathematics subject classification (Amer. Math. Soc.): 16 A 08, 16 A 14, 16 A 52.

\section{Introduction and definitions}

In [6] a ring $R$ was defined to be an extended semihereditary ring if $M(R)$, the left flat epimorphic hull of $R$, is a regular ring, and if every finitely generated right $R$-submodule of $M(R)$ is projective.

A. Hattori [10] defined a right $R$-module to be torsion-free if, for all $a \in A$ and $x \in R, a x=0$ implies that there exist elements $\left\{x_{1}, x_{2}, \ldots, x_{n}\right\} \subseteq R$ with $x_{i} x=0$ for all $1 \leqslant i \leqslant n$, and $\left\{a_{1}, a_{2}, \ldots, a_{n}\right\} \subseteq A$ such that $a=\sum_{i=1}^{n} a_{i} x_{i}$. In [4], this class of modules, which will be denoted by $\mathscr{S}_{R}$, was shown to be the torsion-free class of a perfect torsion theory if and only if $R$ is a right P.P. ring, $M(R) \in \mathscr{S}_{R}$, and $M(R)$ is a regular ring. When this occurs the corresponding perfect topology $G$ is the set of all right ideals of $R$ which contain a finitely generated right essential ideal. This torsion theory will be denoted $\left(\mathscr{T}_{G}, \mathscr{F}_{G}\right)$. The reader is referrred to [7], [13] and [17] for details of torsion theories.

It is shown in Section 2 of this paper that the class of right flat modules is the torsion-free class of a hereditary torsion theory if and only if $R$ is an extended

(C) 1986 Australian Mathematical Society $0263-6115 / 86 \$ A 2.00+0.00$ 
semihereditary ring. When the flat modules do satisfy this property they coincide with $\mathscr{F}_{G}$ and hence with $\mathscr{S}_{R}$.

The category $\mathscr{V}$ of regular rings and ring homomorphisms is shown to be a full, coreflective subcategory of $\mathscr{C}$, the category of extended semihereditary rings with homomorphisms, the ring homomorphisms which induce a left and right flat $R$-module structure, i.e. a ring homomorphism $\phi: R \rightarrow S$ is a homomorphism of $\mathscr{C}$ if it induces a right and left flat $R$-module structure on $S$.

In Section 3 it is shown that for $R$ an extended semihereditary ring, the torsion theory $\left(\mathscr{T}_{G}, \mathscr{F}_{G}\right)$ is the meet, in the lattice of torsion theories, of a class of prime torsion theories. The relationship between these prime torsion theories and the structure of the ring is investigated.

It is shown that each of these prime torsion theories is a perfect maximal torsion theory if and only if $M(R)$, the left flat epimorphic hull of $R$, has artininan primitive factors.

Throughout this paper $R$ is an associative ring with identity, $Q_{\max }(R)$ is the maximal right quotient ring of $R$, and $M(R)$ is the left flat epimorphic hull of $R$. $\mathscr{M}_{R}$ denotes the category of right $R$-modules. If $A \in \mathscr{M}_{R}$, let $E_{R}(A)$ be the right injective hull of $A$. If $X \subseteq R$, let $r_{R}(X)=\{y \in R: X y=0\}$, and let $l_{R}(X)$ be defined similarly. If $I$ is a right ideal of $R$ and $a \in R$, let $\left(I:{ }_{R} a\right)=\{x: a x \in I\}$. The reader is referred to [7] for details of non-singular rings.

$\tau$ will always denote the torsion radical of the torsion theory $\left(\mathscr{T}_{G}, \mathscr{F}_{G}\right)$, and for $A \in \mathscr{M}_{R}, \tau(A)$ will denote the torsion submodule of $A$. The localisation of $A$ with respect to this torsion theory will be denoted by $Q_{\tau}(A)$. A module $A \in \mathscr{M}_{R}$ will be said to be $G$-injective if every diagram of the form

$$
\begin{aligned}
& 0 \rightarrow N^{\prime} \rightarrow N \\
& \alpha \downarrow \measuredangle \beta \quad N / N^{\prime} \in \mathscr{T}_{G} \\
& A
\end{aligned}
$$

can be completed commutatively. The $G$-injective hull of $A \in \mathscr{M}_{R}$ will be denoted by $E_{G}(A)$. A right ideal of an extended semihereditary ring is said to be $G$-closed if $\tau(R / J)=0$.

\section{Extended semihereditary rings}

From Theorem 4.4 of [4] and Theorem 3.3 of [5] the following theorem is obtained.

THEOREM A. For a ring $R$ with maximal right quotient ring $Q_{\max }(R)$, the following are equivalent:

(i) $M(R)$, the left flat epimorphic hull of $R$, is regular, and every finitely generated right $R$-submodule of $M(R)$ is projective. 
(ii) $R$ is right semihereditary, and $Q_{\max }(R)$ is right flat as an $R$-module.

(iii) $R$ is right semihereditary, and every finitely generated non-singular right $R$-module is flat.

(iv) $R$ is left semihereditary, and every finitely generated non-singular left $R$-module is flat.

(v) $\mathscr{S}_{R}$ is the torsion-free class of a perfect torsion theory, and every $A \in \mathscr{S}_{R}$ is a right flat $R$-module.

Following [5], rings satisfying the equivalent conditions of the Theorem will be called extended semihereditary rings.

THEOREM 2.1. The following are equivalent for a ring $R$.

(i) The class of right flat $R$-modules is the torsion-free class of a hereditary torsion theory.

(ii) $R$ is an extended semihereditary ring.

(iii) The class of right flat $R$-modules is the torsion-free class of a perfect torsion theory.

Proof. (i) $\Rightarrow$ (ii). Every submodule of a right flat $R$-module is flat, and hence, the weak global dimension of $R$ is at most 1 . Direct products of flat $R$-modules are flat, and thus $R$ is right semihereditary. $Q_{\max }(R)$ is the injective hull of $R$ and is thus flat. Condition (ii) of Theorem A now implies that $R$ is an extended semihereditary ring.

(ii) $\Rightarrow$ (iii). This is immediate from condition (v) of Theorem A.

(iii) $\Rightarrow$ (i). This is immediate.

The left symmetric conditions corresponding to (i) and (iii) are also equivalent to $R$ being an extended semihereditary ring. This theorem strengthens the result of Cheatham and Enochs [2].

'Extended semihereditary' is a Morita invariant property. Let $R$ be an extended semihereditary ring. Then

(i) If $e$ is an idempotent of $R$ such that $R e R=R$, then $e R e$ is an extended semihereditary ring, and $e M(R) e$ is the left flat epimorphic hull of $e \operatorname{Re}$ [18],

(ii) $(M(R))_{(n)}$, the ring of $n \times n$ matrices over $M(R)$, is the left flat epimorphic hull of $R_{(n)}$, the ring of $n \times n$ matrices over $R$.

EXAMPLES. (i) Every commutative semihereditary ring is an extended semihereditary ring.

(ii) A semiprime semihereditary (left and right) $P I$ ring is an extended semihereditary ring.

(iii) A right semihereditary ring $R$ such that $R_{R}$ is finite dimensional and the maximal right and left quotient rings coincide is an extended semihereditary ring. 
Definition 2.2. A right $R$-module $A_{R}$ is said to be semihereditary if every finitely generated right $R$-module is projective.

THEOREM 2.3. The following are equivalent for a ring $R$ with left flat epimorphic hull $M(R)$ :

(i) $R$ is an extended semihereditary ring;

(ii) $M(R)$ is regular, and the $G$-injective hull, $E_{G}(P)$, of every projective right $R$-module is a semihereditary $R$-module;

(iii) $M / J M$ is a semihereditary $R$-module for each finitely generated right ideal $J$ of $R$.

Proof. (i) $\Leftrightarrow$ (ii). Let $P$ be a projective $R$-module. Then $P \in \mathscr{F}_{G}$, and $E_{G}(P)$ may be considered as an $M(R)$-module. $E_{G}(P)$ is the module of quotients of $P$, and Proposition 17.12 of Golan [6] gives that $E_{G}(P)$ is a projective $M(R)$-module.

Since $M(R)$ is regular, every finitely generated $M$-submodule of $E_{G}(P)$ is projective as an $M(R)$-module. Let $P^{\prime}$ be a finitely generated $R$-submodule of $P$. Then $P^{\prime}$ is flat, and $P^{\prime} \otimes_{R} M$ is a projective submodule of $P \otimes_{R} M \cong E_{G}(P)$. Theorem (3.1) of [10] implies that $P^{\prime}$ is projective. The converse is immediate.

(ii) $\Rightarrow$ (iii). If $J$ is a finitely generated right ideal of $R$, then $J M$ is a direct summand of $M(R)$, as $M(R)$ is regular. Thus $M / J M \cong(1-e) M$ for some idempotent $e \in R$ [6, Theorem 2.2], and $M / J M \doteq E_{G}((1-e) R)$. Thus $M / J M$ is a semihereditary $R$-module.

(iii) $\Rightarrow$ (i). By the assumption, $M(R)$ is a semihereditary $R$-module, and hence $R$ is a right semihereditary ring.

Let $x \in M(R)$. Then $x I \subseteq R$ for a right ideal $I \in F$, the topology of the perfect torsion theory determined by $M(R)$ [7, Proposition 17.8]. Since the torsion theory is perfect, $I$ can be chosen with finite generating set $\left\{s_{1}, s_{2}, \ldots, s_{n}\right\}$. Let $\left\{x s_{1}, x s_{2}, \ldots, x s_{n}\right\}$ be the generating set of a right ideal $J$ of $R$. Then $J M=x M$.

Consider the $R$-submodule of $M / J M$ generated by $1+J M$. The exact sequence

$$
0 \rightarrow x M \cap R \rightarrow R-(1+J M) R \rightarrow 0
$$

splits, since $(1+J M) R$ is projective. Thus $x M \cap R=e R$ for $e^{2}=e \in R$. Thus $x M=e M$, and $M$ is a regular ring. Theorem A now implies that $R$ is an extended semihereditary ring.

The following is a consequence of Theorem 1.1 of [15].

COROLlary 2.4. The ring $\left(\begin{array}{cc}R & M(R) \\ 0 & 0\end{array}\right)$ is a left semihereditary ring if and only if $R$ is an extended semihereditary ring.

A ring $R$ is said to be right P.P. if every principal right ideal of $R$ is projective. 
Proposition 2.5. Let $R$ be a right P.P. ring, $S$ a ring, and $\phi: R \rightarrow S$ a ring homomorphism. Then the following are equivalent.

(i) ${ }_{R} S$ is torsion-free in the sense of Hattori.

(ii) For all $x, y \in R$ such that $r_{R}(x)=r_{R}(y)$, we have $r_{S}(\theta(x))=r_{S}(\theta(y))$.

(iii) For all $x \in R$, we have $\left[\theta\left(r_{R}(x)\right)\right] S=r_{S}(\theta(x))$.

If $R$ is an extended semihereditary ring, then the following conditions are equivalent to (i), (ii), (iii).

(iv) $S$ is a left flat $R$-module.

(v) If $I, J$ are finitely generated left ideals of $R$, then $r_{R}(I)=r_{R}(J)$ implies that $r_{S}(\theta(I))=r_{S}(\theta(J))$.

(vi) For $I, J$ finitely generated left ideals of $R$, we have $\theta\left(r_{R}(I)\right) S=r_{S}(\theta(I))$.

Proof. (i) $\Rightarrow$ (ii) and (iii) $\Rightarrow$ (i) are immediate.

(ii) $\Rightarrow$ (iii). Clearly, we have $\left[\theta\left(r_{R}(x)\right)\right] S \subseteq r_{S}(\theta(x))$. Conversely, assume that $\theta(x) s=0$, where $s \in S$. Since $R$ is a right P.P. ring, there is an idempotent $e \in R$ such that $r_{R}(x)=r_{R}(1-e)$. Hence $\theta(1-e) s=0$. This implies that $\theta(1) s-$ $\theta(e) s=0$, and hence that $s \in[\theta(e R)] S=\left[\theta\left(r_{R}(x)\right)\right] S$.

(i) $\Leftrightarrow$ (iv). This is a consequence of Theorem $A$.

(iv) $\Rightarrow$ (vi). Let $I$ be a finitely generated left ideal of $R$ and $\left\{x_{1}, x_{2}, \ldots, x_{n}\right\}$ a generating set of $I$. Then $r_{R}(I)=\bigcap_{i=1}^{n} r_{R}\left(x_{i}\right)$. Condition (iii) implies that $\theta\left(r_{R}\left(x_{i}\right)\right) S=r_{S}\left(\theta\left(x_{i}\right)\right)$ for all $x_{i}$ and, as $S$ is left flat, that

$$
\begin{aligned}
\theta\left(r_{R}(I)\right) S & =\theta\left(\bigcap_{i=1}^{n} r_{R}\left(x_{i}\right)\right) S=\bigcap_{i=1}^{n} \theta\left(r_{R}\left(x_{i}\right)\right) S \\
& =\bigcap_{i=1}^{n} r_{S}\left(\theta\left(x_{i}\right)\right)=r_{S}(\theta(I)) .
\end{aligned}
$$

The implications (vi) $\Rightarrow(v)$ and $(v) \Rightarrow$ (i) are straightforward and will be omitted.

Proposition 2.6. Let $R$ be an extended semihereditary ring and $J$ a two-sided ideal of $R$ such that $R / J$ is left and right flat as an $R$-module. Then $R / J$ is an extended semihereditary ring.

Proof. $R / J$ is a left and right semihereditary ring, since this property is preserved by flat epimorphisms. Since $R / J$ is right semihereditary, $Z_{R / J}(R / J)=$ 0 , i.e. $R / J$ is a non-singular $(R / J)$-module. Thus $Q_{\max }(R / J)$ is the $(R / J)$-injective hull of $R / J$, and since $R / J$ is left flat as an $R$-module, $Q_{\max }(R / J)$ is also the $R$-injective hull of $R / J$. 
Thus, as injective hulls of right flat $R$-modules are flat, $Q_{\max }(R / J)$ is right flat as an $R$-module, and hence right flat as an $(R / J)$-module. Theorem $\mathrm{A}$ now implies that $R$ is an extended semihereditary ring.

THEOREM 2.7. Let $R$ be an extended semihereditary ring, $S$ a regular ring, and $\phi:$ $R \rightarrow S$ a ring homomorphism such that ${ }_{R} S$ and $S_{R}$ are flat.

Then $S_{R}$ is right $G$-injective $R$-module. (The left symmetric result also holds.)

Proof. Let $I \in G$, and let $\theta \in \operatorname{Hom}_{R}(I, S)$. Then $\theta$ induces an $S$-homomorphism

$$
I \otimes_{R} S \rightarrow S \otimes_{R} S \rightarrow S,
$$

and, as ${ }_{R} S$ is flat, $I \otimes_{R} S \rightarrow I S$ is an isomorphism.

Since $l_{R}(I)=0$, it follows that $l_{S}(\theta(I))=\theta\left(l_{R}(I)\right) S=0$ (Proposition 2.5). Hence $\theta(I) S$ is a finitely generated dense right ideal of $S$, and $I \otimes_{R} S \hat{=} I S=S$, since $S$ is a regular ring.

Hence $\theta$ induces a map

$$
S \rightarrow S \otimes_{R} S \rightarrow S,
$$

i.e. there exists $\theta^{\prime} \in \operatorname{Hom}_{S}(S, S)$ such that $\theta^{\prime} \phi(x)=\theta(x)$ for all $x \in I$. The map $\theta^{\prime} \phi \in \operatorname{Hom}_{R}(R, S)$. By Proposition (4.1) of [6], $S$ is a right $G$-injective $R$-module.

THEOREM 2.8. Let $R$ be an extended semihereditary ring and $M(R)$ the left flat epimorphic hull of $R$, with $\beta: R \rightarrow M(R)$ being the inclusion map of $R$ in $M(R)$.

For any left and right flat ring homomorphism $\theta: R \rightarrow S$ of $R$ into a regular ring $S$, there exists a ring homomorphism $\bar{\theta}: M(R) \rightarrow S$ such that the diagram

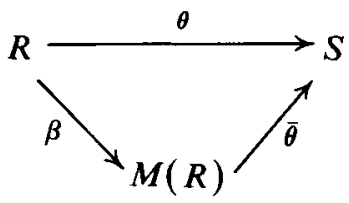

commutes; moreover, the map $\bar{\theta}$ is unique.

Proof. By Theorem 2.7, $S_{R}$ is a $G$-injective $R$-module, is torsion-free in the torsion theory $\left(\mathscr{T}_{G}, \mathscr{F}_{G}\right)$, and hence has the structure of an $M(R)$ module.

Since $M(R) / R \in \mathscr{T}_{G}$, there exists a $\bar{\theta} \in \operatorname{Hom}_{R}(M(R), S)$ such that the diagram

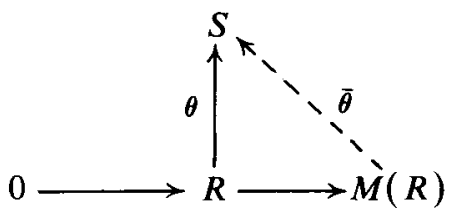


commutes. Since $M(R)$ is a left flat epimorphic image of $R$, the functor $\gamma$ : $\mathscr{M}_{M(R)} \rightarrow \mathscr{M}_{R}$ is full, and $\bar{\theta} \in \operatorname{Hom}_{M}(M(R), S)$. It is straightforward to show that $\bar{\theta}$ is a ring homomorphism and that it is unique.

Let $\mathscr{C}$ denote the category of extended semihereditary rings with homomorphisms being the flat (left and right) ring homomorphisms. Let $\mathscr{V}$ be the category of regular rings with ring homomorphisms. Then $\mathscr{V}$ is a full subcategory of $\mathscr{C}$, and Theorem 2.8 implies that a left adjoint to the inclusion functor exists. Thus we have the following result.

COROLlary 2.9. $\mathscr{V}$ is a full, co-reflective subcategory of $\mathscr{C}$. The map $\beta$ : $R \rightarrow M(R)$ is the left adjoint to the inclusion functor of $\mathscr{V}$ in $\mathscr{C}$.

To conclude this section we present a characterisation of the torsion modules of the torsion theory $\left(\mathscr{T}_{G}, \mathscr{F}_{G}\right)$. The proof is omitted.

Proposition 2.10. For an extended semihereditary ring $R$, we have $A_{R} \in \mathscr{T}_{G}$ if and only if $A \otimes_{R} E=0$ for all left injective $R$-modules $E$.

\section{Prime torsion theories}

The following two results are analogues of classical results for injective modules. They are stated without proof.

LeMma 3.1. Let $(\mathscr{T}, \mathscr{F})$ be a torsion theory for $\mathscr{M}_{R}$ which has maximal closed right ideals. Then if $0 \neq a \in A \in \mathscr{F}$, there exists a maximal closed right ideal $V$ of $R$ and a homomorphism $\phi: A \rightarrow E(R / V)$ such that $\phi(a) \neq 0$.

CorollaRy 3.2. Let $A \in \mathscr{F}$, a torsion-free class of a torsion theory having maximal closed right ideals. Then $A$ may be imbedded in a direct product of modules of the form $E\left(R / V_{\alpha}\right)$ where the $V_{\alpha}$ are the maximal closed right ideals of $R$.

The torsion theory $\left(\mathscr{T}_{G}, \mathscr{F}_{G}\right)$ is a perfect torsion theory of an extended semihereditary ring, and there is a bijection between the right ideals of $M(R)$ and the $G$-closed right ideals of $R$. Each $G$-closed right ideal of $R$ is of the form $J \cap R$ where $J$ is a right ideal of $M(R)$ and $(J \cap R) M(R)=J$ [7, Proposition 17.5].

The torsion theory $\left(\mathscr{T}_{G}, \mathscr{F}_{G}\right)$ has maximal closed right ideals, and these are the contractions of the maximal right ideals of $M(R)$.

Let $\left(\mathscr{T}_{V}, \mathscr{F}_{V}\right)$ be the torsion theory cogenerated by the module $E(R / V)$, [7, page 14], where $V$ is a maximal $G$-closed right ideal of an extended semihereditary 
ring $R$. If $A \in \mathscr{M}_{R}$, let $\tau_{V}(A)$ be the torsion submodule of $A$ with respect to the torsion theory $\left(\mathscr{T}_{V}, \mathscr{F}_{V}\right)$. Let $\left\{V_{\alpha}\right\}$ be the set of all maximal $G$-closed right ideals of $R$.

THEOREM 3.3. For an extended semihereditary ring $R$ :

(i) $\Pi_{\alpha} E\left(R / V_{\alpha}\right)$ is an injective cogenerator of the torsion theory $\left(\mathscr{T}_{G}, \mathscr{F}_{G}\right)$.

(ii) $\left(\mathscr{T}_{G}, \mathscr{F}_{G}\right)$ is the meet, in the lattice of torsion theories, of the torsion theories $\left(\mathscr{T}_{V}, \mathscr{F}_{V}\right)$.

(iii) For each maximal G-closed right ideal $V,\left(\mathscr{T}_{V}, \mathscr{F}_{V}\right)$ is a prime torsion theory.

Proof. (i) This is an immediate consequence of Corollary 3.2.

(ii) This is an immediate consequence of (i) and of the definition of meet [7, page 76 .

(iii) A torsion theory is prime if and only if it is cogenerated by $R / I$, where $I$ is a critical right ideal of $R$ [7, page 196]. Each maximal $G$-closed right ideal of $R$ is a critical right ideal.

Proposition 3.4. Let $S$ be a right ring of quotients of $R$, an extended semihereditary ring such that $S_{R}$ is flat. Then $S$ is an extended semihereditary ring, and ${ }_{R} S$ is flat.

Proof. Let $\phi: R \rightarrow S$. Then $\operatorname{Ker} \phi$ is a right torsion ideal of $R$ and thus $T=R / \operatorname{Ker} \phi$ is left flat as an $R$-module [6, Proposition 2.7]. $T_{R}$ is also flat as $T$ can be considered as a submodule of $S_{R}$ and the weak global dimension of $R$ is at most 1. $T$ is an extended semihereditary ring (Proposition 2.6).

Further, $T \subseteq S \subseteq Q_{\max }(T)$ and $Q_{\max }(T)$ is right and left flat as a $T$-module. The transitivity of flatness gives that $Q_{\max }(T)$ is also left and right flat as an $R$-module. Theorem A now gives that $S$ is an extended semihereditary ring and ${ }_{R} S$ is flat as, the weak global dimension of $R$ is at most 1 .

COROLlaRY 3.5. For an extended semihereditary ring $R$ :

(i) $R_{V}$, the ring of quotients of $R$ with respect to the torsion theory $\left(\mathscr{T}_{V}, \mathscr{F}_{V}\right)$, is right and left flat as an $R$-module.

(ii) $R / \tau_{V}(R)$ is left and right flat as an $R$-module and is an extended semihereditary ring.

Proof. (i) $\mathscr{F}_{V} \subseteq \mathscr{F}_{G}$. Thus $R_{V}$ is right flat as an $R$-module. Proposition 3.4 implies that $R_{V}$ is also left flat.

(ii) This is an easy consequence of (i). 
In Section 2 , the category $\mathscr{C}$ of extended semihereditary rings with left and right flat ring homomorphisms was introduced. In Corollary 3.5 it has been shown that the map $\hat{\tau}_{V}: R \rightarrow R_{V}$ is a homomorphism of $\mathscr{C}$, and that $\tau_{V}(R)$ is a kernel of such a map.

Let $\mathscr{I}$ be the set of ideals of $R$ which are kernels of the homomorphisms of $\mathscr{C}$. In a regular ring every ideal is a member of $\mathscr{I}$. The intersection of all ideals of the form $\tau_{\nu}(R)$ is 0 .

COROLlary 3.6. Let $R$ be an extended semihereditary ring. There is a bijection between the ideals of $M(R)$ and the ideals of $R$ which are members of $\mathscr{I}$ given by $J \rightarrow J \cap R$ and the inverse $I \rightarrow I M(R)$.

Proof. If $x \in J$, a two-sided ideal of $M(R)$, there exist idempotents $e, f \in J$ such that $x=e x$ and $x=x f$. By [6, Theorem 2.2] there exist idempotents $g$ and $h$ of $R$ such that $e M(R)=g M(R)$ and $M(R) f=M(R) h$. Hence $J \cap R \in \mathscr{I}$ by Proposition 2.4 of [6].

Let $J \in \mathscr{I}$. Then $\phi: R \rightarrow R / J$ is a homomorphism of $\mathscr{C}$, and $R / J$ is an extended semihereditary ring (Proposition 2.6). Let $M(R / J)$ be the left flat epimorphic hull of $R / J$. Then there is a map $\phi^{\prime}: M(R) \rightarrow M(R / J)$ such that the diagram

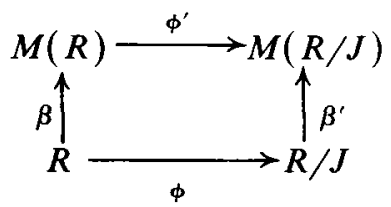

commutes. Now $J=\operatorname{Ker} \phi=\operatorname{Ker} \phi^{\prime} \cap R$. The proof may be concluded by using the properties of the closed ideals of a perfect torsion theory.

Proposition 3.7. Let $R$ be an extended semihereditary ring, and let $J \in \mathscr{I}$. Then $J \subseteq V$, where $V$ is a maximal $G$-closed right ideal of $R$, and furthermore $J \subseteq \tau_{V}(R)$.

Proof. $J$ is a $G$-closed right ideal of $R$ and is thus contained in a maximal $G$-closed right ideal $V$ of $R$.

Let $x \in J \backslash \tau_{V}(R)$. Then there exists $\phi \in \operatorname{Hom}_{R}(x R, E(R / V))$ such that $\phi \neq 0$. Since $E_{R}(R / V)$ is an essential right extension of $R / V$, we have $\operatorname{Im} \phi \cap$ $R / V \neq \varnothing$. Thus there is an element $r \in R$ such that $\phi(x r)=z+V \neq 0+V$. If $y \in r_{R}(x r)$, then $0=\phi(x r y)=z y+V$. Thus $y \in r_{R}(z+V)$, and so it has been shown that $r_{R}(x r) \subseteq r_{R}(z+V)$.

Since $R$ is right semihereditary, there exists $e^{2}=e \in R$ such that $r_{R}(x r)=e R$ and $l_{R} r_{R}(x r)=R(1-e) \subseteq J$. Hence $z=z(1-e)+z e \in V$, and this is a contradiction. Thus $J \subseteq \tau_{V}(R)$. 
COROLlaRY 3.8. If $R$ is a regular ring, and if $P$ is a right primitive ideal of $R$ such that $P=\{x: R x \subseteq V\}$, where $V$ is a maximal right ideal of $R$, then $P=\tau_{V}(R)$.

LEMMA 3.9. Let $R$ be an extended semihereditary ring with left flat epimorphic hull $M(R)$. If $V^{\prime}$ is a maximal right ideal of $M(R)$, and if $V^{\prime} \cap R=V$, then

$$
\tau_{V}(R)=\tau_{V^{\prime}}(M) \cap R .
$$

Each of the ideals $\tau_{V}(R)$ is the contraction of a right primitive ideal of $M(R)$. If every right primitive ideal of $M(R)$ is maximal (e.g. if $M(R)$ is also biregular) then the $\tau_{v}(R)$ are the maximal elements of $\mathscr{I}$.

COROLlaRY 3.10. Let $R$ be an extended semihereditary ring, $V^{\prime}$ a maximal right ideal of $M(R)$, and $V=V^{\prime} \cap R$ the corresponding maximal $G$-closed right ideal of $R$.

Then $M / \tau_{V^{\prime}}(M)$ is the left flat epimorphic hull of $R / \tau_{V}(R)$. Moreover, $R / \tau_{V}(R)$ is an extended semihereditary ring, and $M / \tau_{V}(M)$ is a right primitive regular ring.

Proof. That $R / \tau_{V}(R)$ is a right essential submodule of $M / \tau_{V^{\prime}}(M)$ is a consequence of Lemma 3.9 and the fact that $\tau_{V}(R)$ is $G$-closed. Corollary 3.5 implies that $R / \tau_{V}(R)$ is an extended semihereditary ring, and thus $M / \tau_{V^{\prime}}(M)$ is left and right flat as an $R$-module.

Finally, to prove that $\psi: R / \tau_{V}(R) \rightarrow M / \tau_{V}(M)$ is a ring epimorphism, consider the diagram

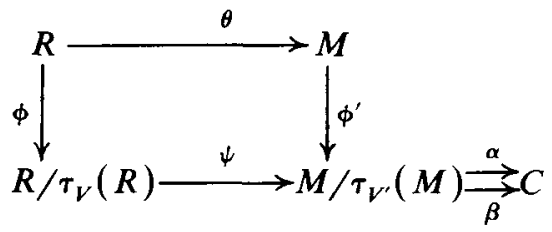

where $C$ is an arbitrary ring, and where $\alpha, \beta$ are ring homomorphisms such that $\alpha \psi=\beta \psi$. The rectangle is commutative, and $\phi^{\prime} \theta$ is a ring epimorphism. Thus $\psi \phi$ is a ring epimorphism, and it is now easily shown that $\psi$ is a ring epimorphism.

COROLlaRY 3.11. Every extended semihereditary ring is a subdirect product of indecomposable extended semihereditary rings.

When $R$ is an extended semihereditary ring and $M(R)$ is biregular, every ideal of $\mathscr{I}$ is generated by central idempotents (the Boolean algebra $B(R)$ of central idempotents of $R$ coincides with $B(M(R))$ ). The ideals of the form $\tau_{V}(R)$ are contractions of the primitive ideals of $M(R)$, and each ideal $\tau_{V}(R)$ is generated 
by the idempotents of a maximal ideal of $B(R)$; that is, $\tau_{V}(R)=x R$, where $x$ is a maximal ideal of $B(R) . R$ can be represented as a Pierce ring $\Gamma(X, \mathscr{R})$ of global sections, where $X=\operatorname{Spec}(B(R))$, the Stone space of the Boolean algebra $B(R)$, and where the Pierce stalks $R_{x}, x \in X$, are indecomposable extended semihereditary rings for which the flat epimorphic hulls are simple regular rings ([3], [16]).

THEOREM 3.12. Let $R$ be an extended semihereditary ring. Then the following are equivalent.

(i) $R_{V}$ is a simple artinian ring for all maximal $G$-closed right ideals of $R$.

(ii) $M(R)$ has artinian primitive factors.

(iii) $\left(\mathscr{T}_{V}, \mathscr{F}_{V}\right)$ is a perfect maximal torsion theory for all maximal $G$-closed right ideals of $R$.

Proof. (i) $\Rightarrow$ (ii). $R_{V}$ being simple artinian implies that $R_{V}$ is the left flat epimorphic hull of $R / \tau_{V}(R)$. Thus, by Corollary 3.10, we have $R_{V} \hat{=} M / \tau_{V^{\prime}}(M)$. Hence $M / \tau_{V}(M)$ is a simple artinian ring.

(ii) $\Rightarrow$ (iii). $R_{V} \hat{=} M / \tau_{V^{\prime}}(M)$ for all $V$, and thus the torsion theories are saturated and perfect. Theorem 17.19 of [7] now gives the result.

(iii) $\Rightarrow$ (i). Again this is a consequence of Theorem 17.19 of [7].

Regular rings with artinian primitive factors are discussed in Chapter 6 of [9].

LEMMA 3.13. Let $R$ be an extended semihereditary ring and $V$ a maximal $G$-closed right ideal of $R$ which is also a member of $\mathscr{I}$. Then $R_{V}$ is a division ring and is the right classical quotient ring of $R / V$.

Proof. $V$ is a right critical ideal of $R$. So Theorem 18.7 of [7] implies that $R / V$ is a right ore domain. Moreover, $\tau_{V}(R)=\bigcap_{f \in \operatorname{Hom}_{R}(R, E(R / V))} \operatorname{Ker} f$, and as $V$ is a right torsion ideal, $\operatorname{Hom}_{R}(V, E(R / V))=0$ [12, Section 2]. Hence $V=\tau_{V}(R)$.

As $V$ is a critical right ideal, $\operatorname{Hom}_{R}\left(R / I, E_{R}(R / V)\right)=0$ for all right ideals $I \supseteq V$. Hence $R_{V}$ is the maximal right quotient ring of $R$.

THEOREM 3.14. Let $R$ be an extended semihereditary ring. Then the following are equivalent.

(i) $\tau_{V}(R)=V$ for all $V$.

(ii) $R_{V}$ is a division ring for all $V$.

(iii) $M(R)$ is strongly regular.

Proof. (i) $\Rightarrow$ (ii). If $\tau_{V}(R)=V$, then $V \in \mathscr{I}$, and Lemma 3.13 gives the result.

(ii) $\Rightarrow$ (iii). $R_{V} \cong M / \tau_{V^{\prime}}(M)$, since $M / \tau_{V^{\prime}}(M)$ is the left flat epimorphic hull of $R / \tau_{V}(R)$. Hence $\tau_{V^{\prime}}(M)$ is a completely prime ideal. Thus $M(R)$ has no non-zero nilpotents and so is strongly regular. 
(iii) $\Rightarrow$ (i). If $M(R)$ is strongly regular, then every ideal of $M(R)$ is two-sided. Thus every $G$-closed right ideal of $R$ is two-sided. Hence, if $V$ is a maximal $G$-closed right ideal of $R$, then $V$ is a contraction of a maximal ideal of $R$. Thus $V \in \mathscr{I}$, and Proposition 3.7 implies that $V=\tau_{V}(R)$.

COROLlaRY 3.15. For an extended semihereditary ring $R$, the following are equivalent.

(i) $\tau_{V}(R)$ is a critical right ideal of $R$ for all maximal $G$-closed right ideals $V$.

(ii) $R / \tau_{V}(R)$ is a right ore domain for all maximal G-closed right ideals $V$.

(iii) $\tau_{V}(R)$ is a completely prime ideal for all maximal $G$-closed right ideals $V$.

(iv) $R$ has no non-zero nilpotents.

(v) $M(R)$ is strongly regular.

In [6] it was shown that an extended semihereditary ring without non-zero nilpotents has a (right and left) classical ring of quotients.

The following examples demonstrate the severity of the conditions necessary for $M(R)$ to be the classical quotient ring of $R$. Further examples appeared in [5].

Examples. 1 [11, Proposition 2]. Let $R$ be a right semihereditary semiprime P.I. ring which is finitely generated as a module over its centre. Then $R$ is an extended semihereditary ring and has a classical quotient ring (right and left) which is a regular ring.

2. Let $R=\left(\begin{array}{c}T T \\ 0\end{array}\right)$, where $T$ is a commutative regular ring. Then $R$ is extended semihereditary ring with left flat epimorphic hull $M(R)=\left({ }_{T T}^{T T}\right)$, and $M(R)$ is a regular biregular ring with artinian primitive factors. $R$ is its own classical right quotient ring.

3. Let $T$ be a right self-injective simple regular ring which is not artinian. Then there exists a maximal right essential ideal $V$ of $T$ which is not two-sided, and $R=\{t \in T: t V \subseteq V\}$ is an extended semihereditary ring which has $T$ as its left flat epimorphic hull [6].

(a) $V \cap R$ is a two-sided ideal of $R$ which is right $G$-closed but which is not a member of $\mathscr{I}$.

(b) $\tau_{V}(R)=0$ for all maximal $G$-closed right ideals $V$ of $R$.

(c) $R$ is semiprime and is its own right classical quotient ring.

When $R$ is a commutative semihereditary ring, then $M(R)$ is the classical quotient ring of $R$. The maximal $G$-closed right ideals of $R$ are the minimal prime ideals of $R$. If $V$ is a maximal ideal of $M(R)$, then $V \cap R=P$ is a minimal prime ideal of $R$, and $M / V=Q_{\mathrm{CL}}(R / P)$, where $R / P$ is a Prüfer domain and $Q_{\mathrm{CL}}(R / P)$ the classical quotient ring. The ideals of the form $\tau_{V}(R)$ are the minimal prime ideals of $R$. 
Many properties of minimal prime ideals in a commutative semihereditary ring have been presented in [14].

In a commutative semihereditary ring each prime ideal $P$ of $R$ contains a unique minimal prime ideal $P^{\prime}$, and

$$
R / P^{\prime} \subseteq R_{P} \subseteq R_{P^{\prime}},
$$

where $R_{p}$ and $R_{P}$, are the rings of quotients of $R$, in the classical sense, with respect to these prime ideals. The following result shows that this desirable property may be generalised to an arbitrary extended semihereditary ring.

Proposition 3.16. Let $R$ be an extended semihereditary ring and $\sigma$ a prime torsion radical such that $\tau_{V}(A) \supseteq \sigma(A)$ for all $A \in \mathscr{M}_{R}$. Then

(i) $\sigma(R)=\tau_{V}(R)$.

(ii) $R / \tau_{V}(R) \subseteq R_{\sigma} \subseteq R_{V}$, where $R_{\mathrm{a}}$ is the ring of quotients corresponding to the torsion radical $\sigma$.

(iii) $R_{\sigma}$ is a left and right flat $R$-module and an extended semihereditary ring.

Proof. Since $\tau_{V}$ and $\sigma$ are prime radicals and $\tau_{V} \geqslant \sigma$, Proposition 19.4 of [6] implies that there is a $\tau_{V}$-critical ideal $I$ and a $\sigma$-critical ideal $I^{\prime}$ such that $\left(I:{ }_{R} a\right) \subseteq\left(I^{\prime}:{ }_{R} a^{\prime}\right)$ for some $a \notin I, a^{\prime} \notin I^{\prime}$. Let $\left(I^{\prime}:_{R} a^{\prime}\right)=I^{\prime \prime}$, which is also a $\sigma$-critical ideal.

Clearly $\sigma(R) \subseteq \tau_{V}(R) \subseteq V$. Let $x \in \tau_{V}(R) \backslash \sigma(R)$. Then

$$
\operatorname{Hom}_{R}\left(x R, E_{R}\left(R / I^{\prime \prime}\right)\right) \neq 0,
$$

and thus there exists $r \in R$ and $z \notin I^{\prime \prime}$ such that $r_{R}(x r) \subseteq r_{R}\left(z+I^{\prime \prime}\right)$, i.e. $z r_{R}(x r) \subseteq I^{\prime \prime}$.

Since $R$ is semihereditary, there exists $e^{2}=e \in R$ such that $r_{R}(x r)=e R$. Now $x r \in \tau_{V}(R)$, and so $l_{R} r_{R}(x r) \subseteq \tau_{V}(R)$ [6, Proposition 2.7]. Hence $R(1-e) \subseteq$ $\tau_{V}(R) \subseteq\left(I:{ }_{R} a\right) \subseteq\left(I^{\prime}: a^{\prime}\right)=I^{\prime \prime}$, and so $z=z e+z(1-e) \in I^{\prime \prime}$, which is a contradiction. Thus $\sigma(R)=\tau_{V}(R)$.

Facts (ii) and (iii) are consequences of (i).

\section{Almost hereditary rings}

Definition 4.1. A module $A_{R}$ is said to be finite injective if, for every $f: I \rightarrow A$ from any finitely generated right ideal, there exists a map $g$ such that the diagram

commutes.

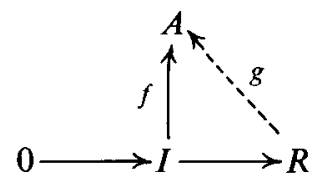


Every injective $R$-module is finite injective, and for a right noetherian ring the two concepts coincide.

Proposition 4.2. If $R$ is a Prüfer domain, then $A_{R}$ is finite injective if and only if it is divisible.

The proof of Proposition 4.2 is a slight adaption of the proof for Dedekind domains that a module is injective if and only if divisible.

'Finite injective' is used as a suitable concept for 'divisibility' in this section. It is evident that for an integral domain, a divisible module is not necessarily finite injective.

The following proposition has a proof which is a slight adaption of the classical result for hereditary rings.

PROPOSITION 4.3. $R$ is a right semihereditary ring if and only if quotients of finite injective $R$-modules are finite injective.

Proposition 4.4 (Eklof and Sabbagh [5]). For a ring $R$, the following are equivalent.

(i) $R$ is regular.

(ii) Every right $R$-module is finite injective.

ProOF. (i) $\Rightarrow$ (ii). Every finitely generated right ideal of a regular ring is a direct summand.

(ii) $\Rightarrow$ (i). $R$ is right semihereditary because quotients of finite injective modules must be finite injective.

Let $x \in R$. If $y \in I_{R} r_{R}(x)$, there is a well-defined map $f: x R \rightarrow y R$ given by $x r \rightarrow y r$. By the assumption, $f$ may be extended to a map $f^{\prime}: R \rightarrow y R$. Assume that $f^{\prime}(1)=a$. Then $f(x r)=a x r=y r$, and in particular $y=a x$. Thus $R x=$ $l_{R} r_{R}(x)$. Since $R$ is a right semihereditary ring, we have $r_{R}(x)=e R$ and $R x=$ $l_{R}(e R)=R(1-e)$ whenever $e^{2}=e \in R$. Thus $R$ is a regular ring.

The proof of (ii) $\Rightarrow$ (i) uses a technique which is also used in Proposition 2.1 of [17].

Definition 4.5. A ring $R$ is right almost hereditary if $R$ is an extended semihereditary ring, and if every right ideal $I \in G$ is projective.

When $R$ is a commutative non-singular ring, the ideals of $G$ are precisely those right ideals of $R$ which are not contained in any minimal prime ideal of $R$.

Thus, is the commutative case, Definition 4.6 coincides with that of Vasconce$\operatorname{los}[20]$. 
Proposition 4.6. For an extended semihereditary ring $R$, every $G$-injective $R$-module is finite injective.

Proof. Let $A_{R}$ be a $G$-injective $R$-module, $I$ a finitely generated right ideal of $R$, and $\operatorname{Hom}_{R}(I, A)$. If $I \in G$, there exists a map $\beta$ such that the following diagram commutes:

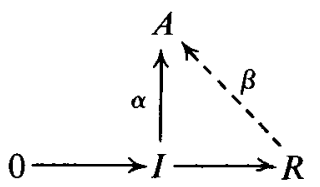

If $I \notin G$, then there exists $e^{2}=e \in R$ such that $I \subseteq e R$ as an essential right submodule. Let $\left\{x_{1}, x_{2}, \ldots, x_{n}\right\}$ be a generating set for $I$ and consider the right ideal $J$ of $R$ which has generating set $x_{1}, x_{2}, \ldots, x_{n}, 1-e$. If $q J=0$ for some $q \in R$, then $q \in R(1-e) \cap R e=0$. Hence $J$ is a finitely generated dense right ideal of $R$, and $J=I \oplus(1-e) R$.

Thus if $\alpha: I \rightarrow A$, there is a map $\alpha^{\prime}: J \rightarrow A$ which extends $\alpha$. By the assumption, there is a map $\beta: R \rightarrow A$ which extends $\alpha^{\prime}$, and hence $\alpha$.

THEOREM 4.7. For an extended semihereditary ring $R$ the following are equivalent.

(i) $R$ is a right almost hereditary ring.

(ii) Every finite injective $R$-module is $G$-injective.

(iii) Every quotient of a G-injective $R$-module is G-injective.

(iv) Every $G$-dense submodule $P^{\prime}$ of a projective $R$-module $P$ is projective (i.e. when $P^{\prime} / P \in \mathscr{T}_{G^{\prime}}$, then $P^{\prime}$ is projective).

Proof. (i) $\Rightarrow$ (ii). If $I \in G$, then $I$ contains a finitely generated dense right ideal. So Proposition 5.19 of [8] implies that $I$ is finitely generated.

(ii) $\Rightarrow$ (iii). This is a consequence of Proposition 4.7 and Proposition 4.3.

(iii) $\Rightarrow$ (iv). Let $P^{\prime}$ be a $G$-dense submodule of a projective $R$-module $P_{R}$. Consider the diagram

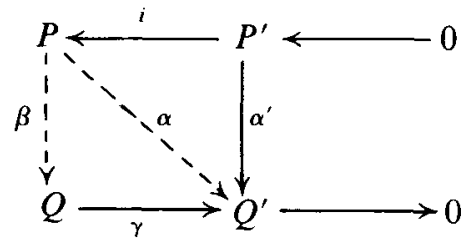

where $Q$ is an injective $R$-module. By the assumption, $Q^{\prime}$ is a $G$-injective $R$-module, and hence there is a map $\alpha: P \rightarrow Q^{\prime}$ such that $\alpha i=\alpha^{\prime}$. Since $P$ is projective, there is a map $\beta: P \rightarrow Q$ such that $\alpha=\gamma \beta$. Thus the rectangle commutes, and so $P^{\prime}$ is projective. 
COROLlaRy 4.8. Let $R$ be an extended semihereditary ring such that $R_{R}$ is finite dimensional. Then the following are equivalent:

(i) $R$ is a right noetherian right hereditary ring;

(ii) $R$ is a right almost hereditary ring.

In the proof of (i) $\Rightarrow$ (ii) of the theorem it was shown that every $I \in G$ is finitely generated. Theorem 3.4 of [6] now implies that every $I \in G$ is invertible in $M(R)$ in the sense of Stenström [17]. This is a further equivalent condition to those of the Theorem 4.8 .

'Right almost hereditary' is a Morita invariant property. There are many examples of right almost hereditary rings which are not right hereditary. Every regular ring is trivially a right almost hereditary ring.

\section{References}

[1] J. Beachy, 'On maximal torsion radicals', Canad. J. Math. 25 (1973), 712-726.

[2] T. Cheatham and E. Enochs, 'Injective hulls of flat modules', Comm. Algebra 8 (20) (1980), 1989-1995.

[3] J. Dauns and K. H. Hofmann, 'The representation of biregular rings by sheaves', Math. Z. 91 (1966), 103-123.

[4] P. Eklof and G. Sabbagh, 'Model-completion and modules', Ann. Math. Logic 2 (1971), 251-295

[5] M. W. Evans, 'Extensions of semi-hereditary rings', J. Austral. Math. Soc. (Series A) 23 (1977), 333-339.

[6] M. W. Evans, 'Extended semi-hereditary rings', J. Austral. Math. Soc. (Series A) 26 (1978), 465-474.

[7] J. S. Golan, Localisation in non-commutative rings (Marcel Dekker Inc., New York, 1974).

[8] K. R. Goodearl, Ring theory (Marcel Dekker Inc., New York, 1976).

[9] K. R. Goodearl, Von Neumann regular rings (Pitman, San Francisco, 1979).

[10] A. Hattori, 'A foundation of torsion theory for modules over general rings', Nagoya Math. J. 17 (1960), 147-158.

[11] S. Jøndrup, 'On finitely generated flat modules II', Math. Scand. 27 (1970), 105-112.

[12] S. Jøndrup, 'Rings of quotients of some semiprime P.I. rings', Comm. Algebra 7(3) (1979), $279-286$.

[13] J. Lambek, 'Torsion theories, additive semantics and rings of quotients', Lecture Notes in Mathematics 177, Springer-Verlag, Berlin (1971).

[14] E. Matlis, 'The minimal prime spectrum of a reduced ring', Illinois J. Math. 27 (1983), 353-391.

[15] A. Page, 'Sur les anneaux hereditaires ou demi-hereditaires', Comm. Algebra 6 (11) (1978), $1169-1186$.

[16] R. S. Pierce, 'Modules over commutative regular rings', Mem. Amer. Math. Soc. 70 (1967).

[17] B. Stenström, Rings of quotients (Springer-Verlag, Berlin, 1975).

[18] H. Storrer, 'Epimorphic extensions of non-commutative rings', Comment Math. Helv. 48 (1973), $72-86$. 
[19] D. Turnbridge, 'Torsion theories and rings of quotients of Morita equivalent rings', Pacific $J$. Math. 37 (1971), 225-234.

[20] W. Vasconcelos, 'Finiteness of projective ideals', J. Algebra 25 (1973), 269-278.

Department of Mathematics

St. Michael's Grammar School

Redan Street

St. Kilda, Victoria 3182

Australia 